\title{
Convergence Estimates for a Linear Backward Euler Scheme for the Saturation Equation
}

\author{
Koffi B. Fadimba*
}

Dept. Math. Sciences, University of South Carolina Aiken, 471 University Parkway, Aiken, SC 29801, USA

\begin{abstract}
In a previous work, stability and consistency results were established for a linearized Euler scheme for the saturation equation. In this paper we continue the mathematical analysis of the scheme, in preparation for its numerical treatment in a future work. We use the regularity results, obtained previously, to establish error estimates in $L^{2}(\Omega)$ for the linear scheme. This work is done with the degenerate nature of the saturation equation in mind, but it is also valid for the non degenerate case like the concentration equation. We show that, if the regularization parameter $\beta$ and the spatial discretization parameter $h$ are carefully chosen in terms of the time stepping parameter $\Delta t$, the convergence is at least of order $O\left((\Delta t)^{\alpha}\right)$ for some determined $\alpha>0$. Examples of choices of $\beta$ and $h$ are given. We also establish a new (at our knowledge) regularity result for the continuous Galerkin formulation of the Saturation Equation and a new regularity result for the linear scheme.
\end{abstract}

2000 Mathematics Subject Classification. 35Q35, 65M06, 65M15, 65M60.

Keywords: Error analysis, linearization, porous medium, nonlinear scheme, degenerate equation, saturation equation, regularity results.

\section{INTRODUCTION}

In considering a numerical approximation of the saturation problem

$$
\begin{cases}\varphi \frac{\partial S}{\partial t}+\nabla \cdot(f(S) u)-\nabla \cdot(k(S) \nabla S)=Q(S) & \text { on } \Omega \times\left(0, T_{0}\right] \\ (f(S) u-k(S) \nabla S) \cdot n=q & \text { on } \Omega \times\left[0, T_{0}\right] \\ S(x, 0)=S^{0}(x) & \text { on } \Omega\end{cases}
$$

obtained from modeling a two-phase immiscible flow through a porous medium [1-4], where $\Omega$ is a bounded domain of $\mathbb{R}^{n}, n=1,2,3$, one encounters two major problems: the nonlinearity of the equation and the degeneracy of the diffusion coefficient $k$. The degeneracy is often addressed by regularizing the problem in some way, though some works bypass this step (see [5]). One then produces a numerical scheme which, often, is still nonlinear. So a linearization in some sense follows in order to implement the scheme on a computer. Two earlier papers [6, 7] dealt with the latter part of the problem. This work is a sequel of these papers.

In (1.1), the unknown $S$ is the saturation of the invading phase. The diffusion coefficient $k$ (see Fig. 1) is the conductivity of the medium and is assumed to satisfy the following conditions.

$$
k(0)=k(1)=0
$$

*Address correspondence to this author at the Dept. Math. Sciences, University of South Carolina Aiken, 471 University Parkway, Aiken, SC 29801, USA; Fax: (803) 641 3726; E-mail: KoffiF@usca.edu

$$
k(\xi) \geq \begin{cases}c_{1} \xi^{\mu} & \text { if } 0 \leq \xi \leq \alpha_{1} \\ c_{2} & \text { if } \alpha_{1}<\xi<\alpha_{2} \\ c_{3}(1-\xi)^{\mu} & \text { if } \alpha_{2} \leq \xi \leq 1,\end{cases}
$$

where $0<\alpha_{1}<\frac{1}{2}<\alpha_{2}<1$, and $0<\mu \leq 2$.

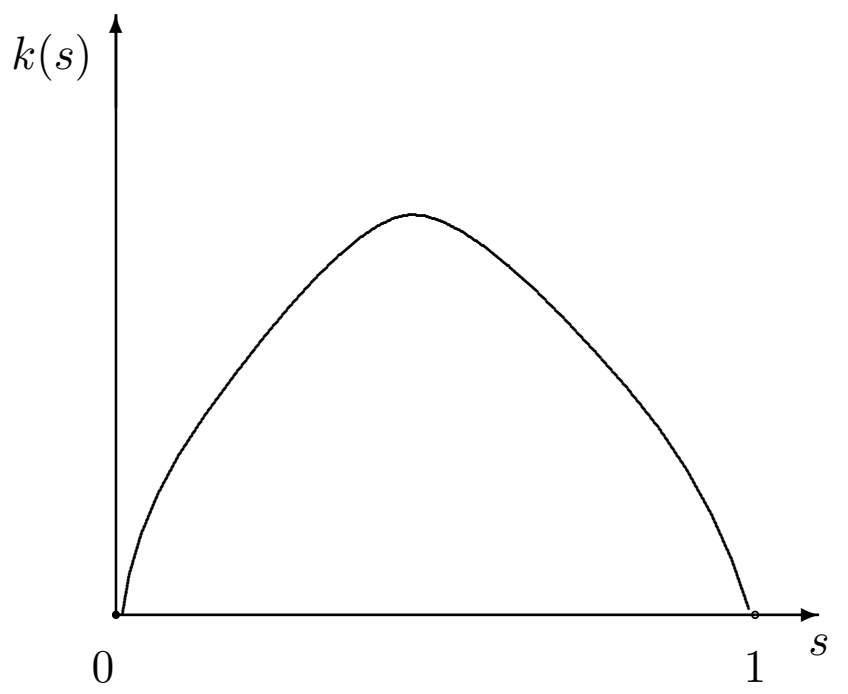

Fig. (1). Example of a graph of $k(s)$.

By [8] and [9], we have

$$
\left|K\left(s_{2}\right)-K\left(s_{1}\right)\right|^{2} \leq C\left|K\left(s_{2}\right)-K\left(s_{1}\right) \| s_{2}-s_{1}\right|
$$

thanks to the fact that $K$ is Lipschitz $\left(K^{\prime}(s)=k(s)\right)$, and

$$
\left|s_{2}-s_{1}\right|^{1+\mu} \leq C\left|K\left(s_{2}\right)-K\left(s_{1}\right)\right| \text {. }
$$


For the derivation of the error estimates, we assume that $k$ is continuously differentiable in the variable $s$.

The function $f$ is twice continuously differentiable in the variable $s$, and

$f^{\prime}(0)=f^{\prime}(1)=0$.

We notice, by [8] and [9], that if (1.3) and (1.6) hold, then

$\left|f\left(s_{2}\right)-f\left(s_{1}\right)\right|^{2} \leq C\left|K\left(s_{2}\right)-K\left(s_{1}\right) \| s_{2}-s_{1}\right|$

where

$$
K(s)=\int_{0}^{s} k(\tau) d \tau .
$$

We also notice, through (1.7) and (1.8), that

$$
\left|f^{\prime}(s)\right| \leq C \sqrt{k(s)} \text {. }
$$

We assume that the porosity of the medium, $\varphi$, satisfies the condtion

$0<\varphi_{0} \leq \varphi(x, t)=\varphi(x) \leq \varphi_{1}<\infty$.

As in [8-10] and [6], we assume that the Darcy velocity $u$ is given and has the necessary regularity we need for this analysis.

The main purpose of this paper is to establish error estimates for a linearization of the Backward Euler scheme obtained by fully discretizing Problem 1.1 (see [6]) Problem 1.1 has been studied by many authors under various forms and conditions (see for instance [8-15], among others). Also similar studies have been done in [16-19] for the Richard's Equation which models water flow (single phase flow) through a porous medium, among other physical phenomena. We also refer to [20] which, in addition to our concern here, includes the study of problems with phase change (Stefan type problems). However, our approach in this work, is different from the approaches in the cited papers in the sense described below.

Because of the degeneracies $(k(0)=k(1)=0)$, Problem 1.1 has often been regularized into a family of nondegenerate problems whose solutions converge to the solution of (1.1) (see $[8-10,12,13,15])$. Usually, the numerical approximation of the solution of (1.1) is done in three steps: regularization, continuous Galerkin method, and fully discretized Galerkin method. In the last step, some of the works cited above obtain a nonlinear implicit scheme (backward Euler). Therefore, one needs to linearize in some way for a computer implementation of the scheme. Often, a Picard iteration is used (see for instance [21] and [16]). For the third step (Discrete Galerkin Method), we choose, in this paper, to linearize the nonlinear functions intervening in (1.1) by Taylor approximations, of first order, of these quantities (see [6]). We then establish error estimates corresponding to this choice.

This paper is a continuation of [6] (where a method was proposed that linearizes the scheme), and of [7] (where some regularity results were established).
The remaining of the paper is structured as follow.

In section 2, we state some preliminary results established in previous works. We establish a new (at least at our knowledge) regularity result for the continuous Galerkin Method. The is helpful in the derivation of error estimates for the linear scheme.

In section 3, we state and prove our second main result, after establishing a regularity result for the linear scheme. Error estimates are obtained through a choice of $\beta$ (the regularization parameter) and/or $h$ (the spatial discretization parameter) in terms of the time-stepping parameter $\Delta t$.

Finally, we set additional notation which will be used throughout the remainder of this paper. We define $(f, g):=(f, g)_{\Omega}:=\int_{\Omega} f g d x$ when this has a meaning. The notation $\|f\|_{L^{p}}:=\|f\|_{L^{p}(\Omega)}$ is used for the standard Lebesgue norm of a measurable function, when this quantity is finite. Similarly, we denote by $\|f\|_{L^{p}\left(L^{q}\right)}:=\|f\|_{L^{p}\left(0, T, L^{q}(\Omega)\right)}$ the mixed Lebesgue norm for $f$. We will also denote $L^{2}\left(t^{n}, t^{n+1}, L^{2}(\Omega)\right):=L^{2}\left(\Omega \times\left[t^{n}, t^{n+1}\right]\right)$. We use $C, c$, to denote positive constants which may change from line, but which are independent of the parameters $\beta, h$ and $\Delta t$, unless otherwise explicitly specified. For $u, v \geq 0$, the notation $u \approx v$ means there exist positive constants $c_{1}$ and $c_{2}$ such that $c_{1} v \leq u \leq c_{2} v$.

\section{PRELIMINARY RESULTS}

In this section, we summarize previous results that are useful for the present analysis and establish a new regularity result.

\subsection{The Regularized Problem}

In order to get a family of nondegenerate problems approximating (1.1), we replace $k$ by $k_{\beta}$, with $k_{\beta} \rightarrow k$ strongly as $\beta \rightarrow 0$ strongly, and with $k_{\beta}$ satisfying the condition:

$0<k_{\beta}(s) \leq\|k\|_{L^{\infty}}=\sup \{k(s), s \in[0,1]\}$.

For example, let $0<\beta \leq \frac{1}{2}$ and define $k_{\beta}$ by

$\delta=\min (k(\beta), k(1-\beta))$.

Define $k_{\beta}$ by

$\begin{cases}k_{\beta}(s)=k(s) & \text { if } k(s) \geq \delta \\ \frac{1}{2} \delta \leq k_{\beta}(s) \leq \delta & \text { otherwise. }\end{cases}$

(see Fig. 2) Set

$K_{\beta}(s)=\int_{0}^{s} k_{\beta}(\tau) d \tau$.

Then, for each $\beta$, we get the nondegenerate problem 


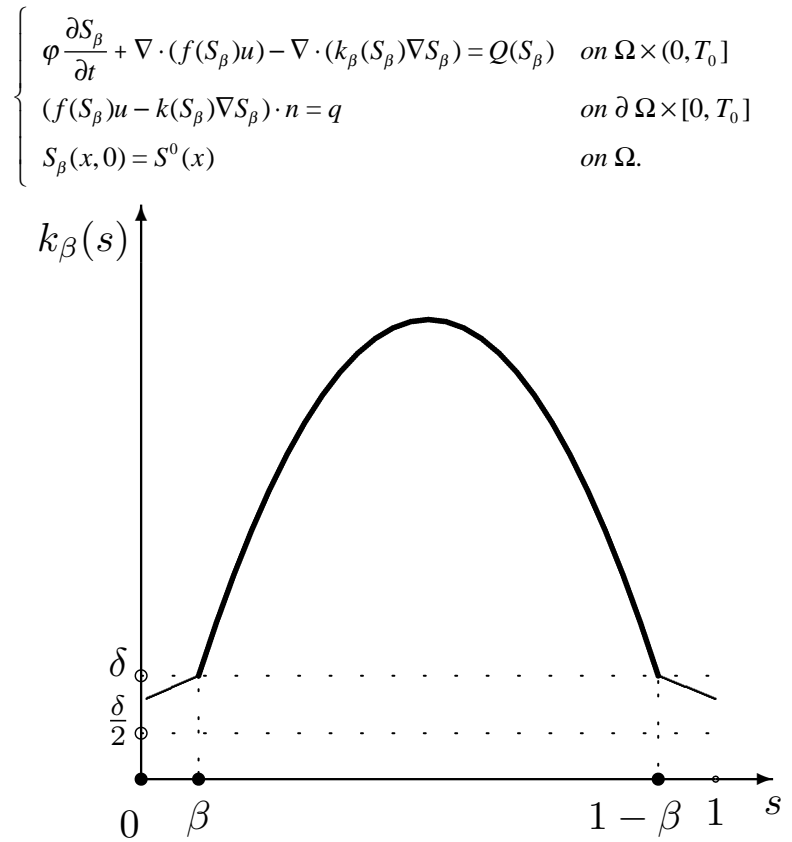

Fig. (2). Example of a perturbation of $k$.

For the remaining of this paper, we assume, to simplify, that $Q \equiv 0$ and $q \equiv 0$. Also, because of (1.10), we can assume, without lost of generality, that $\varphi \equiv 1$.

Let

$C_{0}(\beta)=\left\|K_{\beta}(\cdot)-K(\cdot)\right\|_{L^{\infty}}^{\gamma}$

where

$\gamma=\frac{2+\mu}{1+\mu}$

is the conjugate of $2+\mu$. Let $S$ and $S_{\beta}$ be solutions of (1.1) and (2.5) respectively. Then, by [9],

$\left\|K_{\beta}\left(S_{\beta}\right)-K(S)\right\|_{L^{2}\left(L^{2}\right)} \leq C \beta^{2+\mu}$.

\subsection{Continuous Galerkin Method}

Let $\left\{M_{h}\right\}_{h>0}$ be a family of finite dimensional spaces, with $M_{h} \subset H^{1}(\Omega)$, and assume that $M_{h}$ has the approximation property:

$\inf _{\chi \in M_{h}}\|f-\chi\|_{L^{p}(\Omega)} \leq C h^{2}|f|_{W^{2, p}} \quad$ for all $f \in W^{2, p}(\Omega)$. (2.9)

We will also need the inverse estimate assumption on $M_{h}$ (see, for example, Section 4.5 of [22]):

$\|\chi\|_{H^{1}} \leq C h^{-1}\|\chi\|_{L^{2}} \quad$ for all $\chi \in M_{h}$.

To account for possible numerical oscillations, extend $k_{\beta}$ as follows (and call it again $k_{\beta}$ ):

$k_{\beta}(\xi)= \begin{cases}k_{\beta}(1) & \text { if } \xi \geq 1 \\ k_{\beta}(-\xi) & \text { if } \xi \leq 0 .\end{cases}$
For the same reason, extend the fractional function $f$ as follows.

$f(\xi)= \begin{cases}f(1), & \text { if } \xi \geq 1 \\ f(-\xi), & \text { if } \xi \leq 0\end{cases}$

Then $K_{\beta}$ is bijective from $\mathbb{R}$ to $\mathbb{R}$. We set

$H_{\beta}=K_{\beta}^{-1}$.

Consider the discretized problem: Find $V_{h} \in M_{h}$ such that

$\left(\frac{\partial}{\partial t} H_{\beta}\left(V_{h}\right), \chi\right)-\left(f\left(H_{\beta}\left(V_{h}\right)\right) u, \nabla \chi\right)+\left(\nabla V_{h}, \nabla \chi\right)=0$

for all $\chi \in M_{h}$, and $t \in\left(0, T_{0}\right]$ with the initial condition:

$P_{h} H_{\beta}\left(V_{h}(0)\right)=P_{h} S^{0}$

where $S^{0}$ is as in (1.1), and $P_{h}$ the $L^{2}$ projection on $M_{h}$. $V_{h}$ is hopefully the Galerkin approximation to $K(S)$ with $S$ the solution to Problem 1.1. Indeed, by [10], we have

$\left\|V_{h}-K(S)\right\|_{L^{2}\left(L^{2}\right)} \leq C\left(h^{2 \gamma} \beta^{\frac{-\mu}{1+\mu}}+\beta^{2+\mu}\right)$,

where

$\gamma=\frac{2+\mu}{1+\mu}$.

Remark 2.1 Differentiating (2.14) with respect to the time variable $t$, we obtain

$\frac{d}{d t}\left(\left(\left(H_{\beta}\left(V_{h}\right)\right)_{t}, \chi\right)-\left(f\left(H_{\beta}\left(V_{h}\right)\right) u, \nabla \chi\right)+\left(\nabla V_{h}, \nabla \chi\right)\right)=0$

for all $\chi \in M_{h}$ and for all $t \in[0, T]$.

Because we will be using Taylor expansions of up to order 2 in this analysis, we assume that the solution, $V_{h}$, to (2.14) and (2.15) is three times differentiable in the time variable $t$.

\subsection{Regularity Results for the Continuous Galerkin Method}

The following regularity result was established in [7] and will be useful in this paper. We give a note on an improved proof of this lemma.

Lemma 2.2 Let $V_{h}$ be the solution to problem (2.14)(2.15). Then

$\left\|V_{h t}\right\|_{L^{\infty}\left(L^{2}\right)} \leq C(u) \delta^{-3} h^{-2}$.

\section{Remark 2.3 Note on the proof of the Lemma 2.2.}

The proof of this lemma in [7] seemed to assume that $\left\|V_{h t}\right\|_{L^{4}\left(L^{4}\right)} \leq C$

independently of $\beta$ and $h$. In fact we do not need this assumption. Using the last term on the righthand side of Equation (3.6) of [7], we can write 
$\left|\left(\sqrt{H_{\beta}^{\prime}\left(V_{h}\right)} V_{h t},\left(\sqrt{H_{\beta}^{\prime}\left(V_{h}\right)}\right) V_{t}\right)\right|$

$\leq\left\|\sqrt{H_{\beta}^{\prime}\left(V_{h}\right)} V_{h t}\right\|_{L^{2}}\left\|\left(\sqrt{H_{\beta}^{\prime}\left(V_{h}\right)} V_{h t}\right)_{t}\right\|_{L^{2}}$

$\leq C\left\|\sqrt{H_{\beta}^{\prime}\left(V_{h}\right)} V_{h t}\right\|_{L^{\infty}\left(L^{2}\right)}\left\|H_{\beta}^{\prime \prime}\left(V_{h}\right) V_{h t}^{2}\right\|_{L^{2}}$

where we use the fact that

$$
\left(\sqrt{H_{\beta}^{\prime}\left(V_{h}\right)}\right)_{t}=\frac{1}{2} \frac{H_{\beta}^{\prime \prime}\left(V_{h}\right)}{\sqrt{H_{\beta}^{\prime}\left(V_{h}\right)}} V_{h t} .
$$

With this different manipulation of the terms, Equation (3.14) of [7] becomes

$\frac{1}{2} \frac{d}{d t}\left\|\sqrt{H_{\beta}^{\prime}\left(V_{h}\right)} V_{h t}\right\|_{L^{2}}^{2} \leq C\left\|\sqrt{H_{\beta}^{\prime}\left(V_{h}\right)} V_{h t}\right\|_{L^{\infty}\left(L^{2}\right)}\left\|H_{\beta}^{\prime \prime}\left(V_{h}\right) V_{h t}^{2}\right\|_{L^{2}}$

$+C(u)\left(\nabla V_{h t}\left\|_{L^{2}}^{2}+\frac{1}{\delta}\right\| V_{h t}\left\|_{L^{2}}^{2}+\right\| u_{t} \|_{L^{2}}^{2}\right)$.

Now integrate $(2.21)$ on the interval $(0, t)$, for $t \in\left(0, T_{0}\right)$, and notice that

$\int_{0}^{t}\left\|\sqrt{H_{\beta}^{\prime}\left(V_{h}\right)} V_{h t}\right\|_{L^{\infty}\left(L^{2}\right)}\left\|H_{\beta}^{\prime \prime}\left(V_{h}\right) V_{h t}^{2}\right\|_{L^{2}} d \tau$

$=\left\|\sqrt{H_{\beta}^{\prime}\left(V_{h}\right)} V_{h t}\right\|_{L^{\infty}\left(L^{2}\right)} \int_{0}^{t}\left\|H_{\beta}^{\prime \prime}\left(V_{h}\right) V_{h t}^{2}\right\|_{L^{2}} d \tau$

$\leq \delta^{-3}\left\|\sqrt{H_{\beta}^{\prime}\left(V_{h}\right)} V_{h t}\right\|_{L^{\infty}\left(L^{2}\right)} \int_{0}^{t}\left\|V_{h t}\right\|_{L^{4}}^{2} d \tau$

where we have used (3.51) and the fact that

$\left\|v^{2}\right\|_{L^{2}}=\|v\|_{L^{4}}^{2}$,

for all $v \in L^{4}(\Omega)$. But then,

$\int_{0}^{t}\left\|V_{h t}\right\|_{L^{4}}^{2} d \tau \leq C \int_{0}^{t}\left\|V_{h t}\right\|_{H^{1}}^{2} d \tau$

$\leq C h^{-2} \int_{0}^{t}\left\|V_{h t}\right\|_{L^{2}}^{2} d \tau$

$\leq C h^{-2}\left\|V_{h t}\right\|_{L^{2}\left(L^{2}\right)}^{2}$.

Going back to (2.22), we obtain

$\int_{0}^{t}\left\|\sqrt{H_{\beta}^{\prime}\left(V_{h}\right)} V_{h t}\right\|_{L^{\infty}\left(L^{2}\right)}\left\|H_{\beta}^{\prime \prime}\left(V_{h}\right) V_{h t}^{2}\right\|_{L^{2}} d \tau$

$\leq \sigma_{1}\left\|\sqrt{H_{\beta}^{\prime}\left(V_{h}\right)} V_{h t}\right\|_{L^{\infty}\left(L^{2}\right)}^{2}+\frac{1}{\sigma_{1}} \delta^{-6} h^{-4}\left\|V_{h t}\right\|_{L^{2}\left(L^{2}\right)}^{4}$

Finally take the sup over $[0, T]$ after integrating and then hide the first term of the righthand side of (2.25) in the corresponding term on the left hand side of the final result to get the lemma.

Next, we state and prove a new (at least at our knowledge) regularity result for the Continuous Galerkin Method which is useful in the analysis below. This is the first main result of this paper.
Lemma 2.4 Let $V_{h}$ be the solution to problem (2.14)(2.15). Then

$\left\|\sqrt{H_{\beta}^{\prime}\left(V_{h}\right)} V_{h t t}\right\|_{L^{2}\left(L^{2}\right)}^{2}+\eta\left\|\nabla V_{h t}\right\|_{L^{\infty}\left(L^{2}\right)}^{2} \leq C(u) \delta^{-18} h^{-12}$.

Proof of Lemma 2.4.

In (2.14), set $\chi=V_{h t t}$. Then

$\left(\left(H_{\beta}\left(V_{h}\right)\right)_{t}, V_{h t t}\right)-\left(f\left(H_{\beta}\left(V_{h}\right)\right) u, \nabla V_{h t t}\right)+\left(\nabla V_{h}, \nabla V_{h t t t}\right)=0$.

Let us treat separately each term on the lefthand side of (2.27). Using the product rule, the first term can be written as $\left(\left(H_{\beta}\left(V_{h}\right)\right)_{t}, V_{h t t}\right)=\frac{d}{d t}\left(\left(H_{\beta}\left(V_{h}\right)\right)_{t}, V_{h t t}\right)-\left(\left(H_{\beta}\left(V_{h}\right)\right)_{t t}, V_{h t t}\right)$

$=\frac{d}{d t}\left(\left(H_{\beta}\left(V_{h}\right)\right)_{t}, V_{h t t}\right)$

$-\left(H_{\beta}{ }^{\prime}\left(V_{h}\right) V_{h t t}, V_{h t t}\right)-\left(H_{\beta}{ }^{\prime \prime}\left(V_{h}\right) V_{h t}^{2}, V_{h t t}\right)$

$=\frac{d}{d t}\left(\left(H_{\beta}\left(V_{h}\right)\right)_{t}, V_{h t t}\right)$

$-\left\|\sqrt{H_{\beta}^{\prime}\left(V_{h}\right)} V_{h t t}\right\|_{L^{2}}^{2}-\left(H_{\beta}{ }^{\prime \prime}\left(V_{h}\right) V_{h t}^{2}, V_{h t t}\right)$.

We write the second term on the lefthand side of (2.27) as

$\left(f\left(H_{\beta}\left(V_{h}\right)\right) u, \nabla V_{h t t}\right)=\frac{d}{d t}\left(f\left(H_{\beta}\left(V_{h}\right)\right) u, \nabla V_{h t t}\right)$

$-\left(\left(f\left(H_{\beta}\left(V_{h}\right)\right) u\right)_{t}, \nabla V_{h t t}\right)$.

We rewrite the third term as

$\left(\nabla V_{h}, \nabla V_{h t t}\right)=\frac{d}{d t}\left(\nabla V_{h}, \nabla V_{h t t}\right)-\frac{1}{2} \frac{d}{d t}\left\|\nabla V_{h t}\right\|_{L^{2}}^{2}$

Going back to (2.27) and putting (2.28), (2.29), and (2.30) together, we obtain

$$
\begin{aligned}
& \left\|\sqrt{H_{\beta}{ }^{\prime}\left(V_{h}\right)} V_{h t t}\right\|_{L^{2}}^{2}+\frac{1}{2} \frac{d}{d t}\left\|\nabla V_{h t}\right\|_{L^{2}}^{2} \\
& =\frac{d}{d t}\left(\left(H_{\beta}\left(V_{h}\right)\right)_{t}, V_{h t t}\right)-\left(H_{\beta}{ }^{\prime}\left(V_{h}\right) V_{h t}^{2}, V_{h t t}\right) \\
& -\frac{d}{d t}\left(f\left(H_{\beta}\left(V_{h}\right)\right) u, \nabla V_{h t t}\right) \\
& +\left(\left(f\left(H_{\beta}\left(V_{h}\right)\right) u\right)_{t}, \nabla V_{h t t}\right)+\frac{d}{d t}\left(\nabla V_{h}, \nabla V_{h t t}\right) .
\end{aligned}
$$

Now, by (2.18), if we choose $\chi=V_{h t t}$, we have

$$
\begin{aligned}
& \frac{d}{d t}\left(\left(H_{\beta}\left(V_{h}\right)\right)_{t}, V_{h t t}\right)-\frac{d}{d t}\left(f\left(H_{\beta}\left(V_{h}\right)\right) u, \nabla V_{h t t}\right) \\
& +\frac{d}{d t}\left(\nabla V_{h}, \nabla V_{h t t}\right)=0 .
\end{aligned}
$$

So (2.31) becomes 


$$
\begin{aligned}
& \left\|\sqrt{H_{\beta}^{\prime}\left(V_{h}\right)} V_{h t t}\right\|_{L^{2}}^{2}+\frac{1}{2} \frac{d}{d t}\left\|\nabla V_{h t}\right\|_{L^{2}}^{2} \\
& =-\left(H_{\beta}^{\prime \prime}\left(V_{h}\right) V_{h t}^{2}, V_{h t t}\right) \\
& +\left(\left(f\left(H_{\beta}\left(V_{h}\right)\right) u\right)_{t}, \nabla V_{h t t}\right),
\end{aligned}
$$

which we treat as follows.

$$
\begin{aligned}
& \left\|\sqrt{H_{\beta}{ }^{\prime}\left(V_{h}\right)} V_{h t t}\right\|_{L^{2}}^{2}+\frac{1}{2} \frac{d}{d t}\left\|\nabla V_{h t}\right\|_{L^{2}}^{2} \\
& \leq\left\|\frac{H_{\beta}{ }^{\prime}\left(V_{h}\right) V_{h t}^{2}}{\sqrt{H_{\beta}{ }^{\prime}\left(V_{h}\right)}}\right\|_{L^{2}}^{2}+\frac{1}{4}\left\|\sqrt{H_{\beta}{ }^{\prime}\left(V_{h}\right)} V_{h t t}\right\|_{L^{2}}^{2} \\
& +h^{-2}\left\|\sqrt{\|k\|_{L^{\infty}}}\left(f\left(H_{\beta}\left(V_{h}\right)\right) u\right)_{t}\right\|_{L^{2}}^{2}+\frac{h^{2}}{4}\left\|\frac{1}{\sqrt{\|k\|_{L^{\infty}}}} \nabla V_{h t t}\right\|_{L^{2}}^{2} \\
& \leq\left\|\frac{H_{\beta}{ }^{\prime}\left(V_{h}\right) V_{h t}{ }^{2}}{\sqrt{H_{\beta}{ }^{\prime}\left(V_{h}\right)}}\right\|_{L^{2}}^{2}+\frac{1}{4}\left\|\sqrt{H_{\beta}{ }^{\prime}\left(V_{h}\right)} V_{h t t}\right\|_{L^{2}}^{2} \\
& +h^{-2}\left\|\sqrt{\|k\|_{L^{\infty}}}\left(f\left(H_{\beta}\left(V_{h}\right)\right) u\right)_{t}\right\|_{L^{2}}^{2}+\frac{h^{2}}{4}\left\|\frac{h^{-1}}{\sqrt{\|k\|_{L^{\infty}}}} V_{h t t}\right\|_{L^{2}}^{2} \\
& \leq\left\|\frac{H_{\beta}{ }^{\prime}\left(V_{h}\right) V_{h t}{ }^{2}}{\sqrt{H_{\beta}{ }^{\prime}\left(V_{h}\right)}}\right\|_{L^{2}}^{2}+\frac{1}{4}\left\|\sqrt{H_{\beta}{ }^{\prime}\left(V_{h}\right)} V_{h t t}\right\|_{L^{2}}^{2} \\
& +h^{-2}\left\|\sqrt{\|k\|_{L^{\infty}}}\left(f\left(H_{\beta}\left(V_{h}\right)\right) u\right)_{t}\right\|_{L^{2}}^{2}+\frac{1}{4}\left\|\sqrt{H_{\beta}{ }^{\prime}\left(V_{h}\right)} V_{h t t}\right\|_{L^{2}}^{2}
\end{aligned}
$$$$
\left.\begin{array}{l}
+h^{-2}\left\|\sqrt{\|k\|_{L^{\infty}}}\left(f\left(H_{\beta}\left(V_{h}\right)\right) u\right)_{t} \cdot\right\|_{L^{2}\left(L^{2}\right)}^{2} \\
+\left.\left\|\nabla V_{h t}\right\|_{L^{2}}^{2}\right|_{t=0}
\end{array}\right\}
$$

The first term on the left hand side of (2.37) can be treated as follows.

$$
\begin{aligned}
& \left\|\frac{H_{\beta}{ }^{\prime}\left(V_{h}\right) V_{h t}^{2}}{\sqrt{H_{\beta}{ }^{\prime}\left(V_{h}\right)}}\right\|_{L^{2}}^{2} \leq C \delta^{-6}\left\|V_{h t}^{2}\right\|_{L^{2}}^{2} \leq C \delta^{-6}\left\|V_{h t}\right\|_{L^{4}}^{4} \\
& \leq C \delta^{-6}\left\|V_{h t}\right\|_{H^{1}}^{4} \leq C \delta^{-6} h^{-4}\left\|V_{h t}\right\|_{L^{2}}^{4}
\end{aligned}
$$

where we have used (3.51), the inverse estimate assumption (2.10), and the fact that $H^{1}(\Omega)$ is continuously imbedded in $L^{4}(\Omega)$, for the spatial dimension $n=2$ or $3[23,24]$.

The second term on the left hand side of (2.37) can be treated in the following way.

$$
\begin{aligned}
& h^{-2}\left\|\sqrt{\|k\|_{L^{\infty}}}\left(f\left(H_{\beta}\left(V_{h}\right)\right) u\right)_{t}\right\|_{L^{2}}^{2} \leq C h^{-2}\left\|f^{\prime}\left(H_{\beta}\left(V_{h}\right)\right) H_{\beta}^{\prime}\left(V_{h}\right) V_{h t} u\right\|_{L^{2}}^{2} \\
& +C h^{-2}\left\|f\left(H_{\beta}\left(V_{h}\right)\right) u_{t}\right\|_{L^{2}}^{2} \\
& \leq C h^{-2} \delta^{-1}\|u\|_{L^{\infty}}^{2}\left\|V_{h t}\right\|_{L^{2}}^{2}+C h^{-2}\left\|u_{t}\right\|_{L^{2}}^{2}
\end{aligned}
$$

where we have used (3.52), (1.9), and (2.3). Now by Remark 3.1 of [10],

$\left\|V_{h t}\right\|_{L^{2}\left(L^{2}\right)} \leq C(u)$.

where we have used (2.1), the inverse estimate assumption (2.10), the fact that

$$
H_{\beta}{ }^{\prime}(v)=\frac{1}{k_{\beta}\left(H_{\beta}(v)\right)} \geq \frac{1}{\|k\|_{L^{\infty}}}
$$

by (2.13) and (2.1), and the definition of $k_{\beta},(2.3)$. Also, notice that we have made use of the following fact:

$$
\begin{aligned}
& |a b|=\left|\frac{h a}{\sqrt{2}} \sqrt{2} h^{-1} b\right| \leq \frac{1}{2}\left|\frac{h a}{\sqrt{2}}\right|^{2}+\frac{1}{2}\left|\sqrt{2} h^{-1} b\right|^{2} \\
& \leq \frac{1}{4} h^{2} a^{2}+h^{-2} b^{2}
\end{aligned}
$$

Finally, hide the second and fourth terms on the left hand side of (2.34) in its righthand side and then integrate over $[0, T]$ to obtain

$$
\begin{aligned}
& \left\|\sqrt{H_{\beta}{ }^{\prime}\left(V_{h}\right)} V_{h t t}\right\|_{L^{2}\left(L^{2}\right)}^{2}+\eta\left\|\nabla V_{h t}\right\|_{L^{\infty}\left(L^{2}\right)}^{2} \\
& \leq C\left\{\left\|\frac{H_{\beta}{ }^{\prime}\left(V_{h}\right) V_{h t}^{2}}{\sqrt{H_{\beta}{ }^{\prime}\left(V_{h}\right)}}\right\|_{L^{2}\left(L^{2}\right)}^{2}\right.
\end{aligned}
$$

We also have

$\left\|V_{h t}\right\|_{L^{4}\left(L^{2}\right)}^{4} \leq\left\|V_{h t}\right\|_{L^{\infty}\left(L^{2}\right)}^{4} \leq C \delta^{-12} h^{-8}$,

by Lemma 2.2 .

Therefore, combining (2.37) through (2.39), we get the lemma.

\section{LINEARIZATION AND ERROR ANALYSIS}

In this section, we consider the perturbation given by (2.2) and (2.3) and establish error estimates for the linearized scheme proposed in [6].

\subsection{The Linearized Scheme}

As done in [6], we consider first order Taylor expansions of the functions $H_{\beta}$ and $f \circ H_{\beta}$ :

$$
\begin{aligned}
& H_{\beta}\left(v_{2}\right)-H_{\beta}\left(v_{1}\right)=\left(v_{2}-v_{1}\right) H_{\beta}^{\prime}\left(v_{1}\right)+O\left(\left(v_{2}-v_{1}\right)^{2}\right), \\
& \left(f \circ H_{\beta}\right)\left(v_{2}\right)-\left(f \circ H_{\beta}\right)\left(v_{1}\right)=\left(v_{2}-v_{1}\right)\left(f \circ H_{\beta}\right)^{\prime}\left(v_{1}\right)+O\left(\left(v_{2}-v_{1}\right)^{2}\right) .
\end{aligned}
$$

If we discard the second order terms in (3.1) and (3.2), and replace in (2.14), we obtain the approximate problem:

Find a sequence of functions $\left\{U_{h}^{n}\right\}_{n=0}^{N}$ of $M_{h}$ verifying $\left(\frac{U_{h}^{n+1}-U_{h}^{n}}{\Delta t} H_{\beta}^{\prime}\left(U_{h}^{n}\right), \chi\right)-\left(\left\{\left(f \circ H_{\beta}\right)\left(U_{h}^{n}\right)+\left(U_{h}^{n+1}-U_{h}^{n}\right) \times\right.\right.$ 


$$
\begin{aligned}
& \left.\left.\left(f \circ H_{\beta}\right)^{\prime}\left(U_{h}^{n}\right)\right\} u^{n+1}, \nabla \chi\right) \\
& +\left(\nabla U_{h}^{n+1}, \nabla \chi\right)=0, \quad \forall \chi \in M_{h} \\
& 0 \leq n \leq N-1 \\
& P_{h} H_{\beta} U_{h}^{0}=P_{h} S^{0}
\end{aligned}
$$

where $H_{\beta}$ is defined by (2.13). Here $u^{n}:=u\left(\cdot, t^{n}\right)=u(\cdot, n \Delta t)$.

In this section we wish to show that the solution obtained through this scheme converges to the solution to Problem 1.1 (for $Q=0$ and $q=0$ ) in some functional space. For this, it is enough to show that the solution to Problem (3.3)-(3.4) converges to the solution to (2.14)-(2.15) in that same functional space, independently of $\beta$ and $h$, by $[9,10]$.

Let $A$ be the matrix of the system of linear algebraic equations given by (3.3)-(3.4) (see [6]). The following theorem, which shows the existence and uniqueness for the system above, was proved in [6].

Theorem 3.1 Let $v \in M_{h}$. Then under conditions (1.2)(1.7), we have

$$
\left({ }^{t} v, A v\right) \geq c_{2}\left(1-\frac{\sqrt{\Delta t} c_{3}(u)}{2}\right)\|v\|_{L^{2}(\Omega)}^{2}+\Delta t\|\nabla v\|_{L^{2}(\Omega)}^{2}
$$

where $c_{2}$ and $c_{3}$ are independent of $\beta, h$, and $\Delta t$.

The following regularity result was established in [7] and will be used in the present work

Lemma 3.2 If $\left(U_{h}^{n}\right)_{n=0}^{N} \in M_{h}$ is the solution to the problem (3.3)-(3.4), then

$$
\begin{aligned}
& \sum_{0 \leq n \leq N-1} \Delta t\left\|\sqrt{H_{\beta}^{\prime}\left(U_{h}^{n}\right)} \frac{U_{h}^{n+1}-U_{h}^{n}}{\Delta t}\right\|_{L^{2}}^{2}+\eta \max _{0 \leq n \leq N-1}\left\|\nabla\left(U_{h}^{n+1}\right)\right\|_{L^{2}}^{2} \\
& \leq C(u) \delta^{-1}
\end{aligned}
$$

for some $\eta>0$ and $\delta$ as in (2.2), and $C=C\left(u, U_{h}^{0}, \nabla U_{h}^{0}\right)$

Next we state and prove a new discrete regularity result for the linearized full discretized scheme that will also be helpful in establishing our main result in this paper.

Lemma 3.3 If $\left(U_{h}^{n}\right)_{n=0}^{N} \in M_{h}$ is the solution to the problem (3.3)-(3.4), then

$$
\begin{aligned}
& \max _{0 \leq n \leq N-1}\left\|\frac{U_{h}^{n+1}-U_{h}^{n}}{\Delta t}\right\|_{L^{2}}^{2}+\eta \Delta t \max _{0 \leq n \leq N-1}\left\|\frac{\nabla\left(U_{h}^{n+1}-U_{h}^{n}\right)}{\Delta t}\right\|_{L^{2}}^{2} \\
& \leq C(\Delta t+1) \delta^{-1} h^{-2}
\end{aligned}
$$

for some $\eta>0$ and $\delta$ as in (2.2), and $C=C\left(u, U_{h}^{0}, \nabla U_{h}^{0}\right)$

Proof of Lemma.

Set $\chi=\frac{U_{h}^{n+1}-U_{h}^{n}}{\Delta t}$ in (3.3) to get

$$
\begin{aligned}
& \left\|\frac{U_{h}^{n+1}-U_{h}^{n}}{\Delta t} \sqrt{H_{\beta}^{\prime}\left(U_{h}^{n}\right)}\right\|_{L^{2}}^{2}+\left(\nabla U_{h}^{n+1}, \frac{\nabla\left(U_{h}^{n+1}-U_{h}^{n}\right)}{\Delta t}\right) \\
& =\left(\left(f \circ H_{\beta}\right)\left(U_{h}^{n}\right) u^{n+1}, \frac{\nabla\left(U_{h}^{n+1}-U_{h}^{n}\right)}{\Delta t}\right) \\
& +\left(\left(U_{h}^{n+1}-U_{h}^{n}\right)\left(f \circ H_{\beta}\right)^{\prime}\left(U_{h}^{n}\right) \times\right. \\
& \left.u^{n+1}, \frac{\nabla\left(U_{h}^{n+1}-U_{h}^{n}\right)}{\Delta t}\right) .
\end{aligned}
$$

We split the second term on the lefthand side of (3.8) as follows.

$$
\begin{aligned}
& \left(\nabla U_{h}^{n+1}, \frac{\nabla\left(U_{h}^{n+1}-U_{h}^{n}\right)}{\Delta t}\right)=\Delta t\left\|\frac{\nabla\left(U_{h}^{n+1}-U_{h}^{n}\right)}{\Delta t}\right\|_{L^{2}}^{2} \\
& +\left(\nabla U_{h}^{n}, \frac{\nabla\left(U_{h}^{n+1}-U_{h}^{n}\right)}{\Delta t}\right) .
\end{aligned}
$$

Next, combining (3.8) and (3.9), and using the obvious inequality

$|a b| \leq \frac{1}{2} h^{-2} \sigma^{-1} a^{2}+\frac{1}{2} h^{2} \sigma b^{2}$

by the Young Inequality, we get

$$
\begin{aligned}
& \left\|\frac{U_{h}^{n+1}-U_{h}^{n}}{\Delta t} \sqrt{H_{\beta}^{\prime}\left(U_{h}^{n}\right)}\right\|_{L^{2}}^{2}+\Delta t\left\|\frac{\nabla\left(U_{h}^{n+1}-U_{h}^{n}\right)}{\Delta t}\right\|_{L^{2}}^{2} \\
& \leq C(u)\left\{\sigma^{-1} h^{-2}\left\|\left(f \circ H_{\beta}\right)\left(U_{h}^{n}\right)\right\|_{L^{2}}^{2}\right. \\
& +\sigma^{-1} h^{-2}\left\|\left(f \circ H_{\beta}\right)^{\prime}\left(U_{h}^{n}\right)\left(U^{n+1}-U_{h}^{n}\right)\right\|_{L^{2}}^{2} \\
& \left.+\sigma^{-1} h^{-2}\left\|\nabla U_{h}^{n}\right\|_{L^{2}}^{2}\right\}+\sigma h^{2}\left\|\frac{\nabla\left(U_{h}^{n+1}-U_{h}^{n}\right)}{\Delta t}\right\|_{L^{2}}^{2} .
\end{aligned}
$$

By the regularity assumption on $f$, we obviously have:

$$
\left\|\left(f \circ H_{\beta}\right)\left(U_{h}^{n}\right)\right\|_{L^{2}} \leq C .
$$

The second term on the righthand side of (3.11) can be handled in the following manner.

$$
\begin{aligned}
& \left\|\left(f \circ H_{\beta}\right)^{\prime}\left(U_{h}^{n}\right)\left(U^{n+1}-U_{h}^{n}\right)\right\|_{L^{2}}^{2}= \\
& \left\|f^{\prime}\left(H_{\beta}\left(U_{h}^{n}\right)\right) H_{\beta}^{\prime}\left(U_{h}^{n}\right) \Delta t\left(\frac{U_{h}^{n+1}-U_{h}^{n}}{\Delta t}\right)\right\|_{L^{2}}^{2} \\
& =\left\|f^{\prime}\left(H_{\beta}\left(U_{h}^{n}\right)\right) \sqrt{H_{\beta}^{\prime}\left(U_{h}^{n}\right)} \Delta t\left(\sqrt{H_{\beta}^{\prime}\left(U_{h}^{n}\right)} \frac{U_{h}^{n+1}-U_{h}^{n}}{\Delta t}\right)\right\|_{L^{2}}^{2} \\
& \leq C(\Delta t)^{2}\left\|\frac{U_{h}^{n+1}-U_{h}^{n}}{\Delta t} \sqrt{H_{\beta}^{\prime}\left(U_{h}^{n}\right)}\right\|_{L^{2}}^{2},
\end{aligned}
$$

where we have used (1.9) and the fact that 
$H_{\beta}{ }^{\prime}(s)=\frac{1}{k_{\beta}\left(H_{\beta}(s)\right)}$,

by (2.4) and (2.13). Thus, by Lemma 3.2, we have

$\left\|\left(f \circ H_{\beta}\right)^{\prime}\left(U_{h}^{n}\right)\left(U^{n+1}-U_{h}^{n}\right)\right\|_{L^{2}}^{2} \leq C \Delta t \delta^{-1}$.

Also by the same lemma,

$\left\|\nabla U_{h}^{n}\right\|_{L^{2}}^{2} \leq C \delta^{-1}$.

Thanks to the inverse estimate assumption (2.10), and because $U_{h}^{n+1}-U_{h}^{n} \in M_{h}$, the last term of the righthand side of (3.11) can be treated as follows.

$\left\|\frac{\nabla\left(U_{h}^{n+1}-U_{h}^{n}\right)}{\Delta t}\right\|_{L^{2}}^{2} \leq C h^{-2}\left\|\frac{U_{h}^{n+1}-U_{h}^{n}}{\Delta t}\right\|_{L^{2}}^{2}$.

We also notice that

$H_{\beta}{ }^{\prime}(s) \geq \frac{1}{\|k(\cdot)\|_{L^{\infty}}}$

by (2.1) and (3.14). Hence the first term on the lefthand side of (3.11) is bounded below as follows.

$\left\|\frac{U_{h}^{n+1}-U_{h}^{n}}{\Delta t} \sqrt{H_{\beta}^{\prime}\left(U_{h}^{n}\right)}\right\|_{L^{2}}^{2} \geq\|k(\cdot)\|_{L^{\infty}}^{-1}\left\|\frac{U_{h}^{n+1}-U_{h}^{n}}{\Delta t}\right\|_{L^{2}}^{2}$.

Substituting (3.19) in (3.11), multiplying the new inequality by $\|k(\cdot)\|_{L^{\infty}}$, and using (3.12), (3.13), (3.15), and (3.16), we obtain

$\left\|\frac{U_{h}^{n+1}-U_{h}^{n}}{\Delta t}\right\|_{L^{2}}^{2}+\|k(\cdot)\|_{L^{\infty}} \Delta t\left\|\frac{\nabla\left(U_{h}^{n+1}-U_{h}^{n}\right)}{\Delta t}\right\|_{L^{2}}^{2}$

$\leq C(u) \sigma^{-1} h^{-2}\left(1+\Delta t \delta^{-1}+\delta^{-1}\right)$

$+\sigma\|k(\cdot)\|_{L^{\infty}}\left\|\frac{U_{h}^{n+1}-U_{h}^{n}}{\Delta t}\right\|_{L^{2}}^{2}$.

Now, choose $\sigma$ in the arithmetic-geometric mean inequality (3.10) so that

$\sigma\|k(\cdot)\|_{L^{\infty}} \leq \frac{1}{2}$,

in order to hide the last term on the righthand side of (3.20) in the corresponding term of the lefthand side. Finally, take the maximum of both sides over $0 \leq n \leq N$ to get the Lemma.

\subsection{Error Estimates}

We now establish the second main result of this paper.

\subsubsection{Preparation for the Main Theorem}

To prepare for the statement and the proof of our main results, we do some preliminary work.

Let $V_{h} \in M_{h}$ be the solution to Problem (2.14)-(2.15). Let $V_{h}^{n}:=V_{h}\left(\cdot, t^{n}\right)$ with $t^{n}=n \Delta t$.
Using a first order Taylor expansion of the function $H_{\beta}(v)$ in the variable $v$, we obtain

$$
H_{\beta}\left(v_{2}\right)-H_{\beta}\left(v_{1}\right)=\left(v_{2}-v_{1}\right) H_{\beta}^{\prime}\left(v_{1}\right)+\frac{\left(v_{2}-v_{1}\right)^{2}}{2} H_{\beta}{ }^{\prime}\left(v_{0}\right)
$$

with $v_{0}$ between $v_{1}$ and $v_{2}$. Hence, we have

$$
\begin{aligned}
& \frac{H_{\beta}\left(U_{h}^{n+1}\right)-H_{\beta}\left(U_{h}^{n}\right)}{\Delta t}=\frac{U_{h}^{n+1}-U_{h}^{n}}{\Delta t} H_{\beta}^{\prime}\left(U_{h}^{n}\right) \\
& +\frac{\left(U_{h}^{n+1}-U_{h}^{n}\right)^{2}}{2 \Delta t} H_{\beta}{ }^{\prime \prime}\left(\psi_{h}^{n}\right)
\end{aligned}
$$

for some $\psi_{h}^{n}$ between $U_{h}^{n}$ and $U_{h}^{n+1}$.

Similarly, using a first order Taylor expansion of the function $\left(f \circ H_{\beta}\right)(v)$, we get

$$
\begin{aligned}
& \left(f \circ H_{\beta}\right)\left(U_{h}^{n+1}\right)=\left(f \circ H_{\beta}\right)\left(U_{h}^{n}\right)+\left(U_{h}^{n+1}-U_{h}^{n}\right)\left(f \circ H_{\beta}\right)^{\prime}\left(U_{h}^{n}\right) \\
& +\frac{\left(U_{h}^{n+1}-U_{h}^{n}\right)^{2}}{2}\left(f \circ H_{\beta}\right)^{\prime \prime}\left(\psi_{h}^{n}\right)
\end{aligned}
$$

for some $\varphi_{h}^{n}$ between $U_{h}^{n}$ and $U_{h}^{n+1}$.

We can rewrite (2.14) as

$$
\begin{aligned}
& \left(\frac{H_{\beta}\left(V_{h}^{n+1}\right)-H_{\beta}\left(V_{h}^{n}\right)}{\Delta t}, \chi\right)-\left(\left(f \circ H_{\beta}\right)\left(V_{h}^{n+1}\right) u^{n+1}, \nabla \chi\right) \\
& +\left(\frac{\partial\left(H_{\beta}\left(V^{n}\right)\right)}{\partial t}-\frac{H_{\beta}\left(V_{h}^{n+1}\right)-H_{\beta}\left(V_{h}^{n}\right)}{\Delta t}, \chi\right) \\
& +\left(\nabla V_{h}^{n+1}, \nabla \chi\right)=0, \forall \chi \in M_{h} .
\end{aligned}
$$

Next using (3.24) and (3.23) in (3.3), we get

$$
\left(\frac{H_{\beta}\left(U_{h}^{n+1}\right)-H_{\beta}\left(U_{h}^{n}\right)}{\Delta t}, \chi\right)-\left(\frac{\left(U_{h}^{n+1}-U_{h}^{n}\right)^{2}}{2 \Delta t} H_{\beta}^{\prime \prime}\left(\psi_{h}^{n}\right), \chi\right)
$$$$
-\left(f \circ H_{\beta}\left(U_{h}^{n+1}\right) u^{n+1}, \chi\right)
$$$$
-\left(\frac{\left(U_{h}^{n+1}-U_{h}^{n}\right)^{2}}{2}\left(f \circ H_{\beta}\right)^{\prime \prime}\left(\varphi_{h}^{n}\right) u^{n+1}, \nabla \chi\right)
$$$$
+\left(\nabla U_{h}^{n+1}, \nabla \chi\right)=0, \quad \forall \chi \in M_{h}
$$

$0 \leq n \leq N-1$

Now, subtract (3.25) from (3.26) and rewrite to get

$$
\begin{aligned}
& \left(\frac{H_{\beta}\left(U_{h}^{n+1}\right)-H_{\beta}\left(V_{h}^{n+1}\right)}{\Delta t}-\frac{H_{\beta}\left(U_{h}^{n}\right)-H_{\beta}\left(V_{h}^{n}\right)}{\Delta t}, \chi\right) \\
& +\left(\nabla\left(U_{h}^{n+1}-V_{h}^{n+1}\right), \nabla \chi\right) \\
& -\left(\left(\left(f \circ H_{\beta}\right)\left(U_{h}^{n+1}\right)-\left(f \circ H_{\beta}\right)\left(V_{h}^{n+1}\right)\right) u^{n+1}, \nabla \chi\right)
\end{aligned}
$$




$$
\begin{aligned}
& -\left(\frac{\left(U_{h}^{n+1}-U_{h}^{n}\right)^{2}}{2}\left(f \circ H_{\beta}\right)^{\prime \prime}\left(\varphi_{h}^{n}\right) u^{n+1}, \nabla \chi\right) \\
& -\left(\frac{\partial\left(H_{\beta}\left(V^{n}\right)\right)}{\partial t}-\frac{H_{\beta}\left(V_{h}^{n+1}\right)-H_{\beta}\left(V_{h}^{n}\right)}{\Delta t}, \chi\right) \\
& -\left(\frac{\left(U_{h}^{n+1}-U_{h}^{n}\right)^{2}}{2 \Delta t} H_{\beta}^{\prime \prime}\left(\psi_{h}^{n}\right), \chi\right)=0, \forall \chi \in M_{h} .
\end{aligned}
$$

We need the following for the proof of Theorem 3.4 below.

\subsubsection{The Discrete Analogue of the Poisson Solution Operator}

We give some properties of the discrete analogue of the Poisson operator, which we need in the proof of our second main result below. For more details on this operator, see the appendix of [7], for instance.

We define the operator $T_{h}$ from $\left(H^{1}(\Omega)\right)^{*}$ into $M_{h}$ (defined in section 2) by $T_{h} f=\omega_{h}$, where $\omega_{h}$ is the unique solution of the discretized elliptic problem:

$\left\{\begin{array}{l}\left(\nabla \omega_{h}, \nabla \chi\right)=\left(f-f_{\Omega}, \chi\right) \quad \text { for all } \chi \in M_{h}, \\ \left(\omega_{h}\right)_{\Omega}=f_{\Omega}\end{array}\right.$

where $g_{\Omega}:=\frac{1}{|\Omega|} \int_{\Omega} g(x) d x$, is the mean value of $g$ over $\Omega$. In the present analysis, we assume that $|\Omega|=1$, to simplify.

Then we have the following properties.

$\left(\nabla T_{h} f, \nabla \chi\right)=(f, \chi)-f_{\Omega} \chi_{\Omega}, \forall \chi \in M_{h}$,

$\left(T_{h} \chi, \chi\right) \approx\|\chi\|_{\left(H^{1}(\Omega)\right)^{*}}=:\|\chi\|_{H_{h}^{-1}} \forall \chi \in M_{h}$,

$\left\|\nabla T_{h} \chi\right\|_{L^{2}} \leq\|\chi\|_{H_{h}^{-1}} \forall \chi \in M_{h}$,

and

$\left\|T_{h} \chi\right\|_{L^{2}} \leq\left\|T_{h} \chi\right\|_{H^{1}} \approx\|\chi\|_{H_{h}^{-1}}, \forall \chi \in M_{h}$.

Finally, by definition of $T_{h}$, and by the fact that

$f_{\Omega}=\int_{\Omega} f d x=(f, 1)=\left(P_{h} f, 1\right)=\left(P_{h} f\right)_{\Omega}$,

(since $1 \in M_{h}$ ), we have

$T_{h} f=T_{h} P_{h} f$

for all $f \in\left(H^{1}(\Omega)\right)^{*}$, where $P_{h}$ is the $L^{2}$-projection onto $M_{h}$.

\subsubsection{The Main Theorem}

Thanks to the above preparatory work, we can now state and prove our second main result.

Theorem 3.4 Suppose conditions (1.2)-(1.9) hold. Let $V_{h}$ be the solution to (2.14)-(2.15). Denote
$V_{h}^{n}:=V_{h}\left(\cdot, t^{n}\right)=V_{h}(\cdot, n \Delta t), 0 \leq n \leq N$. Let $\left\{U_{h}^{n}\right\}_{0 \leq n \leq N}$ be the solution to (3.3)-(3.4). Then

$$
\begin{aligned}
& \max _{0 \leq n \leq N-1}\left\|P_{h} H_{\beta}\left(U_{h}^{n+1}\right)-P_{h} H_{\beta}\left(V_{h}^{n+1}\right)\right\|_{H_{h}^{-1}}^{2} \\
& +\eta \sum_{0 \leq n \leq N-1} \Delta t\left(\left(U_{h}^{n+1}-V_{h}^{n+1}\right), H_{\beta}\left(U_{h}^{n+1}\right)-H_{\beta}\left(V_{h}^{n+1}\right)\right) \\
& \leq C(u)\left((\Delta t)^{2}\left(h^{-12} \delta^{-19}\right)\right),
\end{aligned}
$$

for some $\eta>0$.

\section{Proof.}

We first notice that, for $\chi \in M_{h}$, we have

$$
\left(H_{\beta}\left(U_{h}^{n+1}\right)-H_{\beta}\left(V_{h}^{n+1}\right), \chi\right)=\left(P_{h}\left(H_{\beta}\left(U_{h}^{n+1}\right)-H_{\beta}\left(V_{h}^{n+1}\right)\right), \chi\right)
$$

Also, by [7], page 376,

$\frac{1}{2}\left\|P_{h}\left(H_{\beta}\left(U_{h}^{n+}\right)-H_{\beta}\left(V_{h}^{n+1}\right)\right)\right\|_{H_{h}^{-1}}^{2}-\frac{1}{2}\left\|P_{h}\left(H_{\beta}\left(U_{h}^{n}\right)-H_{\beta}\left(V_{h}^{n}\right)\right)\right\|_{H_{h}^{-1}}^{2}$ $\leq\left(P_{h}\left(H_{\beta}\left(U_{h}^{n+1}\right)-H_{\beta}\left(V_{h}^{n+1}\right)\right), T_{h}\left(H_{\beta}\left(U_{h}^{n+1}\right)-H_{\beta}\left(V_{h}^{n+1}\right)\right)\right)$. (3.37)

In (3.27), set $\chi=T_{h}\left(H_{\beta}\left(U_{h}^{n+1}\right)-H_{\beta}\left(V_{h}^{n+1}\right)\right)$, and use (3.32), (3.36), (3.37), and the arithmetic-geometric inequality to obtain

$$
\begin{aligned}
& \frac{1}{2 \Delta t}\left\{\left\|P_{h}\left(H_{\beta}\left(U_{h}^{n+1}\right)-H_{\beta}\left(V_{h}^{n+1}\right)\right)\right\|_{H_{h}^{-1}}^{2}\right. \\
& \left.-\left\|P_{h}\left(H_{\beta}\left(U_{h}^{n}\right)-H_{\beta}\left(V_{h}^{n}\right)\right)\right\|_{H_{h}^{-1}}^{2}\right\} \\
& +\left(\nabla\left(U_{h}^{n+1}-V_{h}^{n+1}\right), \nabla T_{h}\left(H_{\beta}\left(U_{h}^{n+1}\right)-H_{\beta}\left(V_{h}^{n+1}\right)\right)\right. \\
& \leq \sigma_{1}\left\|\left(f\left(H_{\beta}\left(U_{h}^{n}\right)\right)-f\left(H_{\beta}\left(V_{h}^{n}\right)\right)\right) u^{n+1}\right\|_{L^{2}}^{2}+E \\
& +F+G+C\left\|\nabla T_{h}\left(H_{\beta}\left(U_{h}^{n+1}\right)-H_{\beta}\left(V^{n+1}\right)\right)\right\|_{L^{2}}^{2}
\end{aligned}
$$

where $\sigma_{1}$ can be made arbitrary small thanks to the arithmetic-geometric inequality, and where the terms $E, F$, and $G$ are to be specified and treated below.

Using (3.29), the third term on the left side of (3.38), can be treated as follow.

$$
\begin{aligned}
& \left(\nabla\left(U_{h}^{n+1}-V_{h}^{n+1}\right), \nabla T_{h}\left(H_{\beta}\left(U_{h}^{n+1}\right)-H_{\beta}\left(V_{h}^{n+1}\right)\right)\right. \\
& =\left(U_{h}^{n+1}-V_{h}^{n+1}, H_{\beta}\left(U_{h}^{n+1}\right)-H_{\beta}\left(V_{h}^{n+1}\right)\right) \\
& -\left(U_{h}^{n+1}-V_{h}^{n+1}\right)_{\Omega}\left(H_{\beta}\left(U_{h}^{n+1}\right)-H_{\beta}\left(V_{h}^{n+1}\right)\right)_{\Omega} .
\end{aligned}
$$

We rewrite (3.38) using (3.31), (3.34), and (3.39):

$$
\begin{aligned}
& \frac{1}{2 \Delta t}\left\{\left\|P_{h}\left(H_{\beta}\left(U_{h}^{n+1}\right)-H_{\beta}\left(V_{h}^{n+1}\right)\right)\right\|_{H_{h}^{-1}}^{2}\right. \\
& \left.-\left\|P_{h}\left(H_{\beta}\left(U_{h}^{n}\right)-H_{\beta}\left(V_{h}^{n}\right)\right)\right\|_{H_{h}^{-1}}^{2}\right\} \\
& +\left(\left(U_{h}^{n+1}-V_{h}^{n+1}\right),\left(H_{\beta}\left(U_{h}^{n+1}\right)-H_{\beta}\left(V_{h}^{n+1}\right)\right)\right.
\end{aligned}
$$




$$
\begin{aligned}
& \leq\left(U_{h}^{n+1}-V_{h}^{n+1}\right)_{\Omega}\left(H_{\beta}\left(U_{h}^{n+1}\right)-H_{\beta}\left(V_{h}^{n+1}\right)\right)_{\Omega} \\
& +\sigma_{1}\left\|\left(f\left(H_{\beta}\left(U_{h}^{n+1}\right)\right)-f\left(H_{\beta}\left(V_{h}^{n+1}\right)\right)\right) u^{n+1}\right\|_{L^{2}}^{2}+E \\
& +F+G+C\left\|P_{h}\left(H_{\beta}\left(U_{h}^{n+1}\right)-H_{\beta}\left(V^{n+1}\right)\right)\right\|_{H_{h}^{-1}}^{2}
\end{aligned}
$$

Next, we treat each of the terms on the righthand side of (3.40) separately. The first term on the righthand side of (3.40) is treated as follows.

$$
\begin{aligned}
& \left|\left(U_{h}^{n+1}-V_{h}^{n+1}\right)_{\Omega}\left(H_{\beta}\left(U_{h}^{n+1}\right)-H_{\beta}\left(V_{h}^{n+1}\right)\right)_{\Omega}\right| \leq \sigma_{2}\left(U_{h}^{n+1}-V_{h}^{n+1}\right)_{\Omega}^{2} \\
& +\frac{1}{\sigma_{2}}\left(H_{\beta}\left(U_{h}^{n+1}\right)-H_{\beta}\left(V_{h}^{n+1}\right)\right)_{\Omega}^{2} .
\end{aligned}
$$

We have

$$
\begin{aligned}
& \left|\left(U_{h}^{n+1}-V_{h}^{n+1}\right)_{\Omega}\right|=\left|\int_{\Omega}\left(U_{h}^{n+1}-V_{h}^{n+1}\right) d x\right| \\
& \leq|| U_{h}^{n+1}-V_{h}^{n+1} \|_{L^{2}}
\end{aligned}
$$

by Holder Inequality (using the assumption that $|\Omega|=1$ ). In the same way, we have

$$
\begin{aligned}
& \left|\left(H_{\beta}\left(U_{h}^{n+1}\right)-H_{\beta}\left(V_{h}^{n+1}\right)\right)_{\Omega}\right|=\left|\int_{\Omega}\left(H_{\beta}\left(U_{h}^{n+1}\right)-H_{\beta}\left(V_{h}^{n+1}\right)\right) d x\right| \\
& =\left|\int_{\Omega} P_{h}\left(H_{\beta}\left(U_{h}^{n+1}\right)-H_{\beta}\left(V_{h}^{n+1}\right)\right) d x\right| \\
& \leq\left\|P_{h}\left(H_{\beta}\left(U_{h}^{n+1}\right)-H_{\beta}\left(V_{h}^{n+1}\right)\right)\right\|_{H_{h}^{-1}}\|1\|_{H^{1}} \\
& \leq\left\|P_{h}\left(H_{\beta}\left(U_{h}^{n+1}\right)-H_{\beta}\left(V_{h}^{n+1}\right)\right)\right\|_{H_{h}^{-1}},
\end{aligned}
$$

where we have used (3.33).

We can now rewrite (3.41) using (3.42) and (3.43):

$$
\begin{aligned}
& \left|\left(U_{h}^{n+1}-V_{h}^{n+1}\right)_{\Omega}\left(H_{\beta}\left(U_{h}^{n+1}\right)-H_{\beta}\left(V_{h}^{n+1}\right)\right)_{\Omega}\right| \\
& \leq \sigma_{2}\left\|U_{h}^{n+1}-V_{h}^{n+1}\right\|_{L^{2}}^{2} \\
& +C\left\|P_{h}\left(H_{\beta}\left(U_{h}^{n+1}\right)-H_{\beta}\left(V_{h}^{n+1}\right)\right)\right\|_{H_{h}^{-1}}^{2} .
\end{aligned}
$$

The second term on the righthand side of (3.40) is bounded as follows.

$$
\begin{aligned}
& \left\|\left(f\left(H_{\beta}\left(U_{h}^{n+1}\right)\right)-f\left(H_{\beta}\left(V_{h}^{n+1}\right)\right)\right) u^{n+1}\right\|_{L^{2}}^{2} \\
& \leq C\|u\|_{L^{\infty}\left(L^{\infty}\right)}^{2}\left(U_{h}^{n+1}-V_{h}^{n+1}, H_{\beta}\left(U_{h}^{n+1}\right)-H_{\beta}\left(V_{h}^{n+1}\right)\right),
\end{aligned}
$$

by (1.7).

To treat the term

$$
F=\left\|\frac{\partial\left(H_{\beta}\left(V^{n}\right)\right)}{\partial t}-\frac{H_{\beta}\left(V_{h}^{n+1}\right)-H_{\beta}\left(V_{h}^{n}\right)}{\Delta t}\right\|_{L^{2}}^{2},
$$

we notice, using Taylor expansion, that

$$
\begin{aligned}
& \frac{\partial\left(H_{\beta}\left(V^{n}\right)\right)}{\partial t}-\frac{H_{\beta}\left(V_{h}^{n+1}\right)-H_{\beta}\left(V_{h}^{n}\right)}{\Delta t} \\
& =\frac{1}{\Delta t} \int_{t^{n}}^{t^{n+1}}\left(H_{\beta}\left(V_{h}(\cdot, \tau)\right)\right)_{t t}\left(\tau-t^{n}\right) d \tau .
\end{aligned}
$$

Hence, the term $F$ is bounded as follows.

$$
\begin{aligned}
& F=\left\|\frac{1}{\Delta t} \int_{t^{n}}^{t^{n+1}}\left(H_{\beta}\left(V_{h}(\cdot, \tau)\right)\right)_{t t}\left(\tau-t^{n}\right) d \tau\right\|_{L^{2}}^{2} \\
& \leq\left(\frac{1}{\Delta t} \int_{t^{n}}^{t^{n+1}}\left\|\left(H_{\beta}\left(V_{h}\right)\right)_{t t}\right\|\left(\tau-t^{n}\right) d \tau\right)^{2} \\
& \leq \Delta t\left\|\left(H_{\beta}\left(V_{h}\right)\right)_{t t}\right\|_{L^{2}\left(t^{n}, t^{n+1} ; L^{2}(\Omega)\right)}^{2} .
\end{aligned}
$$

To bound the terms $E, F$, and $G$, one can check easily that, for $v \in \mathbb{R}$,

$$
H_{\beta}^{\prime \prime}(v)=\frac{-k_{\beta}^{\prime}\left(H_{\beta}(v)\right)}{\left(k_{\beta}\left(H_{\beta}(v)\right)\right)^{3}} \text {. }
$$

and

$$
\left(f \circ H_{\beta}\right)^{\prime \prime}(v)=\frac{f^{\prime \prime}\left(H_{\beta}(v)\right) k_{\beta}\left(H_{\beta}(v)\right)-f^{\prime}\left(H_{\beta}(v)\right) k_{\beta}^{\prime}\left(H_{\beta}(v)\right)}{\left(k_{\beta}\left(H_{\beta}(v)\right)\right)^{3}}
$$

where we have used (2.4) and (2.13).

Thus, with the assumption that $k$ is continuously differentiable and $f$ twice continuously differentiable, we get

$$
\left|\left(f \circ H_{\beta}\right)^{\prime \prime}(v)\right| \leq \frac{C}{\delta^{3}}
$$

and

$$
\left|\left(H_{\beta}\right)^{\prime \prime}(v)\right| \leq \frac{C}{\delta^{3}},
$$

for all $v \in \mathbb{R}$. Also

$$
\left|H_{\beta^{\prime}}(v)\right|=\left|\frac{1}{k_{\beta}\left(H_{\beta}(v)\right)}\right| \leq \frac{C}{\delta},
$$

by (2.3) and (2.13).

Now

$\left(H_{\beta}\left(V_{h}\right)\right)_{t t}=H_{\beta}^{\prime \prime}\left(V_{h}\right) V_{h t}^{2}+H_{\beta}^{\prime}\left(V_{h}\right) V_{h t}$,

and

$$
\begin{aligned}
& \left\|H_{\beta}^{\prime}\left(V_{h}\right) V_{h t t}\right\|_{L^{2}}=\left\|\sqrt{H_{\beta}^{\prime}\left(V_{h}\right)} \sqrt{H_{\beta}^{\prime}\left(V_{h}\right)} V_{h t t}\right\|_{L^{2}} \\
& \leq \frac{1}{\sqrt{\delta}}\left\|\sqrt{H_{\beta}^{\prime}\left(V_{h}\right)} V_{h t t}\right\|_{L^{2}} .
\end{aligned}
$$

Therefore 


$$
\begin{aligned}
& \left\|\left(H_{\beta}\left(V_{h}\right)\right)_{t t}\right\|_{L^{2}\left(t^{n}, t^{n+1}, L^{2}(\Omega)\right)} \leq\left\|H_{\beta}^{\prime \prime}\left(V_{h}\right) V_{h t}^{2}\right\|_{L^{2}\left(t^{n}, t^{n+1}, L^{2}(\Omega)\right)} \\
& +\frac{1}{\sqrt{\delta}}\left\|\sqrt{H_{\beta}^{\prime}\left(V_{h}\right)} V_{h t t}\right\|_{L^{2}\left(t^{n}, t^{n+1}, L^{2}(\Omega)\right)}
\end{aligned}
$$

where we have used the fact that $H^{1}(\Omega)$ is continuously imbedded in $L^{4}(\Omega)$, for $n=2$ or 3 (see for instance [23, $24]$ ), and (3.58) below.

Hence, (3.47) becomes

$$
\begin{aligned}
& F \leq C \Delta t\left(\left\|H_{\beta}^{\prime \prime}\left(V_{h}\right) V_{h t}^{2}\right\|_{L^{2}\left(t^{n}, t^{n+1}, L^{2}(\Omega)\right)}^{2}\right) \\
& +\frac{1}{\delta}\left\|\sqrt{H_{\beta}^{\prime}\left(V_{h}\right)} V_{h t t}\right\|_{L^{2}\left(t^{n}, t^{n+1}, L^{2}(\Omega)\right)}^{2}
\end{aligned}
$$

The term $E$ is bounded as follows,

$$
\begin{aligned}
& E=\left\|\frac{\left(U_{h}^{n+1}-U_{h}^{n}\right)^{2}}{2}\left(f \circ H_{\beta}\right)^{\prime \prime}\left(\varphi_{h}^{n}\right)\right\|_{L^{2}}^{2} \\
& \leq \frac{C(\Delta t)^{2}}{\delta^{6}}\left((\Delta t)^{2}\left\|\left(\frac{U_{h}^{n+1}-U_{h}^{n}}{\Delta t}\right)^{2}\right\|_{L^{2}}^{2}\right) .
\end{aligned}
$$

\section{Given that}

$\left\|v^{2}\right\|_{L^{2}}=\|v\|_{L^{4}}^{2}$, for all $v \in L^{4}$,

estimate (3.57) becomes

$$
E \leq \frac{C(\Delta t)^{2}}{\delta^{6}}\left(\Delta t\left\|\frac{U_{h}^{n+1}-U_{h}^{n}}{\Delta t}\right\|_{L^{4}}^{2}\right)^{2} .
$$

The term $G$ is treated as the term $E$ and we get:

$$
\begin{aligned}
& G=\left\|\frac{\left(U_{h}^{n+1}-U_{h}^{n}\right)^{2}}{2 \Delta t} H_{\beta}^{\prime \prime}\left(\psi_{h}^{n}\right)\right\|_{L^{2}}^{2} \\
& \leq \frac{C}{\delta^{6}}\left(\Delta t\left\|\frac{U_{h}^{n+1}-U_{h}^{n}}{\Delta t}\right\|_{L^{4}}^{2}\right)^{2} .
\end{aligned}
$$

Combining (3.44), (3.45), (3.56), (3.59), and (3.60) with (3.40), we get

$$
\begin{aligned}
& \frac{1}{2 \Delta t}\left\{\left\|P_{h}\left(H_{\beta}\left(U_{h}^{n+1}\right)-H_{\beta}\left(V_{h}^{n+1}\right)\right)\right\|_{H_{h}^{-1}}^{2}\right. \\
& \left.-\left\|P_{h}\left(H_{\beta}\left(U_{h}^{n}\right)-H_{\beta}\left(V_{h}^{n}\right)\right)\right\|_{H_{h}^{-1}}^{2}\right\} \\
& +\left(\left(U_{h}^{n+1}-V_{h}^{n+1}\right),\left(H_{\beta}\left(U_{h}^{n+1}\right)-H_{\beta}\left(V_{h}^{n+1}\right)\right)\right. \\
& \leq \sigma_{2}\left\|U_{h}^{n+1}-V_{h}^{n+1}\right\|_{L^{2}}^{2} \\
& +\sigma_{1}\|u\|_{L^{\infty}\left(L^{\infty}\right)}^{2}\left(U_{h}^{n+1}-V_{h}^{n+1}, H_{\beta}\left(U_{h}^{n+1}\right)-H_{\beta}\left(V_{h}^{n+1}\right)\right)
\end{aligned}
$$

$$
\begin{aligned}
& +\frac{C(\Delta t)^{2}}{\delta^{6}}\left(\Delta t\left\|\frac{U_{h}^{n+1}-U_{h}^{n}}{\Delta t}\right\|_{L^{4}}^{2}\right)^{2} \\
& +C \Delta t\left(\left\|H_{\beta}^{\prime \prime}\left(V_{h}\right) V_{h t}^{2}\right\|_{L^{2}\left(t^{n}, t^{n+1}, L^{2}(\Omega)\right)}^{2}\right) \\
& \left.+\delta^{-1}\left\|\sqrt{H_{\beta}^{\prime}\left(V_{h}\right)} V_{h t t}\right\|_{L^{2}\left(t^{n}, t^{n+1}, L^{2}(\Omega)\right)}^{2}\right) \\
& +\frac{C}{\delta^{6}}\left(\Delta t \|\left.\frac{U_{h}^{n+1}-U_{h}^{n}}{\Delta t}\right|_{L^{4}} ^{2}\right)^{2} \\
& +C\left\|P_{h}\left(H_{\beta}\left(U_{h}^{n+1}\right)-H_{\beta}\left(V^{n+1}\right)\right)\right\|_{H_{h}^{-1}}^{2} .
\end{aligned}
$$

Next, in view of (1.7), choose, in the arithmeticgeometric inequality, $\sigma_{2}$ such that

$$
\sigma_{2}\left\|U_{h}^{n+1}-V_{h}^{n+1}\right\|_{L^{2}}^{2} \leq \frac{1}{4}\left(U_{h}^{n+1}-V_{h}^{n+1}, H_{\beta}\left(U_{h}^{n+1}\right)-H_{\beta}\left(V_{h}^{n+1}\right)\right) .
$$

In the same manner, choose $\sigma_{1}$ such that

$$
\sigma_{1}\|u\|_{L^{\infty}\left(L^{\infty}\right)}^{2} \leq \frac{1}{4} \text {. }
$$

Then the first two terms on the righthand side of (3.61) can be hidden in its lefthand side. After hiding these terms, multiply (3.61) by $\Delta t$, and then sum for $0 \leq n \leq m$, with $0 \leq m \leq N-1$, to get

$$
\begin{aligned}
& \frac{1}{2}\left\{\left\|P_{h}\left(H_{\beta}\left(U_{h}^{m+1}\right)-H_{\beta}\left(V_{h}^{m+1}\right)\right)\right\|_{H_{h}^{-1}}^{2}\right. \\
& \left.-\left\|P_{h}\left(H_{\beta}\left(U_{h}^{0}\right)-H_{\beta}\left(V_{h}^{0}\right)\right)\right\|_{H_{h}^{-1}}^{2}\right\}
\end{aligned}
$$$$
+\frac{1}{2} \sum_{0 \leq n \leq m} \Delta t\left(\left(U_{h}^{n+1}-V_{h}^{n+1}\right),\left(H_{\beta}\left(U_{h}^{n+1}\right)-H_{\beta}\left(V_{h}^{n+1}\right)\right)\right.
$$$$
\leq C\left(\|u\|_{L^{\infty}\left(L^{\infty}\right)}\right)\left\{\frac{\Delta t^{3}+\Delta t}{\delta^{6}} \sum_{0 \leq n \leq m-1}\left(\Delta t\left\|\frac{U_{h}^{n+1}-U_{h}^{n}}{\Delta t}\right\|_{L^{4}}^{2}\right)^{2}\right.
$$$$
\left.+\Delta t^{2}\left(\left\|H_{\beta}^{\prime \prime}\left(V_{h}\right) V_{h t}^{2}\right\|_{L^{2}\left(L^{2}\right)}^{2}+\delta^{-1}\left\|\sqrt{H_{\beta}^{\prime}\left(V_{h}\right)} V_{h t t}\right\|_{L^{2}\left(L^{2}\right)}^{2}\right)\right\}
$$$$
+C \sum_{0 \leq n \leq m} \Delta t\left\|P_{h}\left(H_{\beta}\left(U_{h}^{n+1}\right)-H_{\beta}\left(V^{n+1}\right)\right)\right\|_{H_{h}^{-1}}^{2} .
$$

The second term on the left hand side of (3.64) vanishes thanks to (2.15) and (3.4).

We notice the following obvious fact.

$$
\sum_{0 \leq i \leq n}\|v\|_{L^{p}\left(t^{i}, t^{i+1}, L^{p}(\Omega)\right)}^{p}=\|v\|_{L^{p}\left(0, T_{0}, L^{p}(\Omega)\right)}^{p}, 1 \leq p<\infty,
$$

for all $v \in L^{p}\left(0, T_{0}, L^{p}(\Omega)\right)$. This justifies the presence of the second and third terms in the righthand side of (3.64).

Next, to deal with the first term in the righthand side of (3.64), we notice the obvious fact: If $\left(a_{i}\right)$ are nonnegative real quantities for $1<i<n$, then 
$\sum_{1 \leq i \leq n} a_{i}^{2} \leq\left(\sum_{1 \leq i \leq n} a_{i}\right)^{2}$

Using this fact, we obtain

$$
\begin{aligned}
& \sum_{0 \leq n \leq m-1}\left(\Delta t\left\|\frac{U_{h}^{n+1}-U_{h}^{n}}{\Delta t}\right\|_{L^{4}}^{2}\right)^{2} \leq\left(\sum_{0 \leq n \leq m-1} \Delta t\left\|\frac{U_{h}^{n+1}-U_{h}^{n}}{\Delta t}\right\|_{L^{4}}^{2}\right)^{2} \\
& \leq\left(\sum_{0 \leq n \leq m-1} \Delta t\left\|\frac{U_{h}^{n+1}-U_{h}^{n}}{\Delta t}\right\|_{H^{1}}^{2}\right)^{2} \\
& \leq C h^{-4}\left(\sum_{0 \leq n \leq m-1} \Delta t\left\|\frac{U_{h}^{n+1}-U_{h}^{n}}{\Delta t}\right\|_{L^{2}}^{2}\right)^{2},
\end{aligned}
$$

where we have used the fact that $H^{1}(\Omega)$ is continuously imbedded in $L^{4}(\Omega)$ and the inverse estimate assumption (2.10). Now, use (2.35) and Lemma 3.3 to see that

$$
\sum_{0 \leq n \leq m-1}\left(\Delta t\left\|\frac{U_{h}^{n+1}-U_{h}^{n}}{\Delta t}\right\|_{L^{4}}^{2}\right)^{2} \leq C(u) T_{0} \Delta t h^{-8} \delta^{-2} .
$$
obtain

Use lemma 2.4, (2.38), (2.41), and (3.68) in (3.64) to

$$
\begin{aligned}
& \frac{1}{2}\left\{\left\|P_{h}\left(H_{\beta}\left(U_{h}^{m+1}\right)-H_{\beta}\left(V_{h}^{m+1}\right)\right)\right\|_{H_{h}^{-1}}^{2}\right. \\
& +\frac{1}{2} \sum_{0 \leq n \leq m} \Delta t\left(\left(U_{h}^{n+1}-V_{h}^{n+1}\right),\left(H_{\beta}\left(U_{h}^{n+1}\right)-H_{\beta}\left(V_{h}^{n+1}\right)\right)\right. \\
& \leq C(u)\left((\Delta t)^{2}\left(h^{-12} \delta^{-19}+h^{-8} \delta^{-8}\right)\right) \\
& +C \Delta t \sum_{0 \leq n \leq m}\left\|P_{h}\left(H_{\beta}\left(U_{h}^{n+1}\right)-H_{\beta}\left(V^{n+1}\right)\right)\right\|_{H_{h}^{-1}}^{2} .
\end{aligned}
$$

Finally, apply the discrete Gronwall Lemma to (3.69) and then take the maximum for $0 \leq m \leq N-1$ to get the theorem.

Remark 3.5 The application of the discrete Gronwall Lemma to (3.69) needs some justification. Split the last term of (3.69) as follows.

$$
\begin{aligned}
& C \Delta t \sum_{0 \leq n \leq m}\left\|P_{h}\left(H_{\beta}\left(U_{h}^{n+1}\right)-H_{\beta}\left(V^{n+1}\right)\right)\right\|_{H_{h}^{-1}}^{2} \\
& =C \Delta t\left\|P_{h}\left(H_{\beta}\left(U_{h}^{m+1}\right)-H_{\beta}\left(V^{m+1}\right)\right)\right\|_{H_{h}^{-1}}^{2} \\
& +C \Delta t \sum_{0 \leq n \leq m-1}\left\|P_{h}\left(H_{\beta}\left(U_{h}^{n+1}\right)-H_{\beta}\left(V^{n+1}\right)\right)\right\|_{H_{h}^{-1}}^{2} .
\end{aligned}
$$

Now, for $\Delta t$ sufficient small so that

$$
\frac{1}{2}-C \Delta t \geq c_{0}>0 \text {, }
$$

for some $c_{0}>0$, the first term on the righthand side of (3.70) can be hidden in the first term of the lefthand side of (3.69).
Theorem 3.4 can be made specific thanks to (1.5) and (1.7). We do this through the following.

Corollary 3.6 Under the conditions of Theorem 3.4, we have

$$
\begin{aligned}
& \sum_{0 \leq n \leq N} \Delta t\left\|U_{h}^{n+1}-V_{h}^{n+1}\right\|_{L^{2}}^{2} \leq C(u)(\Delta t)^{2} h^{-12} \delta^{-19}, \\
& \sum_{0 \leq n \leq N} \Delta t\left\|H_{\beta}\left(U_{h}^{n+1}\right)-H_{\beta}\left(V_{h}^{n+1}\right)\right\|_{L^{2+\mu}}^{2+\mu} \leq C(u)(\Delta t)^{2} h^{-12} \delta^{-19},
\end{aligned}
$$

where $\mu$ is as in (1.3).

Clearly, Theorem 3.4 (through Corollary 3.6) states that, for fixed $\beta$ (thus $\delta$ ), the regularization parameter, and $h$, the spatial discretization parameter, the solution to problem (3.3) and (3.4) converges to the solution to problem (2.14) and (2.15). More interestingly, it also states that for a fixed $\beta$, the solution to problem (3.3) and (3.4) converges to the solution to problem 2.5 uniformly in $h$, if $h$ is appropriately chosen in terms of $\Delta t$. This mean that scheme (3.3)-(3.4), as we expected, will converge faster when applied to the non degenerate case i.e. the case where $k(s) \geq k_{0}>0$, for all $s \in[0,1]$.

\section{CONCRETE EXAMPLES AND DISCUSSION}

We illustrate these through examples of appropriate choices of $\beta$ and $h$ in terms of $\Delta t$.

By (1.3) and (2.2), we have

$\delta \geq c \beta^{\mu}$.

As in $[13,12]$, for the corollary below, we make the assumption

$k(s) \leq c_{3} s^{\mu}$.

We choose $\beta$ and $h$ in terms of $\Delta t$ as follows.

$\beta \approx h^{\lambda_{1}}$,

and

$h \approx(\Delta t)^{\lambda_{2}}$,

with $\lambda_{1}>0$ and $\lambda_{2}>0$.

Define

$\alpha=2-19 \mu \lambda_{1} \lambda_{2}-12 \lambda_{2}$,

\subsection{Choice 1}

In view of (4.3), if we choose $\lambda_{1}$ such that (2.16) becomes

$\left\|V_{h}-K(S)\right\|_{L^{2}\left(L^{2}\right)}=O\left(\beta^{2+\mu}\right)=O\left(h^{\lambda_{1}(2+\mu)}\right)$,

then it suffices that,

$\lambda_{1}=\frac{4+2 \mu}{2+4 \mu+\mu^{2}}$. 
Set

$K^{n}(\cdot):=K\left(S\left(\cdot, t^{n}\right)\right)=K(S(\cdot, n \Delta t))$.

Then, thanks to the triangle inequality, we have the following

Corollary 4.1 Under the conditions of Theorem 3.4, and conditions (4.3) through (4.8), we have

$\sum_{0 \leq n \leq N-1} \Delta t\left\|K^{n}-U_{h}^{n+1}\right\|_{L^{2}\left(L^{2}\right)}^{2} \leq C(u)(\Delta t)^{\alpha}$.

Next we choose $\lambda_{1}$ as in (4.7), with $\mu$ as in (1.3). If we then set

$\lambda_{2}=\frac{3}{4} \frac{2+4 \mu+\mu^{2}}{12+62 \mu+25 \mu^{2}}$,

then

$\alpha=\frac{1}{2}$.

For $\lambda_{1}$ given by (4.7), if we set

$c_{\mu}=\frac{2+4 \mu+\mu^{2}}{12+62 \mu+25 \mu^{2}}$,

we get Table 1. As expected, we see that the rate of convergence $\alpha_{2}$ decreases as $\lambda_{2}$ increases.

Table 1. Variation of the Rate of Convergence as a Function of $\lambda_{2}$

\begin{tabular}{|c|c|c|c|c|c|c|}
\hline \multicolumn{6}{|c|}{$\lambda_{1}=\frac{4+2 \mu}{2+4 \mu+\mu^{2}}$} & \\
\hline$\lambda_{2}$ & $\frac{7 c_{\mu}}{8}$ & $\frac{5 c_{\mu}}{6}$ & $\frac{3 c_{\mu}}{4}$ & $\frac{2 c_{\mu}}{3}$ & $\frac{3 c_{\mu}}{5}$ & $\frac{c_{\mu}}{2}$ \\
\hline$\alpha$ & $\frac{1}{4}$ & $\frac{1}{3}$ & $\frac{1}{2}$ & $\frac{2}{3}$ & $\frac{4}{5}$ & 1 \\
\hline
\end{tabular}

\subsection{Choice 2}

Putting (2.16) and (3.72) together and using the triangle inequality, we get

$$
\begin{aligned}
& \sum_{0 \leq n \leq N-1} \Delta t\left\|K^{n}-U_{h}^{n+1}\right\|_{L^{2}\left(L^{2}\right)}^{2} \leq C(u)\left(\beta^{2+\mu}\right. \\
& \left.+h^{2 \gamma} \beta^{-\frac{\mu}{\mu+1}}+(\Delta t)^{\alpha}\right) .
\end{aligned}
$$

Now, choose $\lambda_{1}$ and $\lambda_{2}$ in such a way that

$$
\sum_{0 \leq n \leq N-1} \Delta t\left\|K^{n}-U_{h}^{n+1}\right\|_{L^{2}\left(L^{2}\right)}^{2}=O\left(\beta^{2+\mu}\right)=O\left((\Delta t)^{\lambda_{1} \lambda_{2}(2+\mu)}\right) .
$$

For this purpose, from (4.11), it suffices that $\left\{\begin{array}{l}2 \gamma \lambda_{2}-\left(\frac{\mu}{1+\mu}\right) \lambda_{1} \lambda_{2}=\lambda_{1} \lambda_{2}(2+\mu) \\ 2-19 \mu \lambda_{1} \lambda_{2}-12 \lambda_{2}=\lambda_{1} \lambda_{2}(2+\mu)\end{array}\right.$

Using (2.7) and solving system (4.13) for $\lambda_{1}$ and $\lambda_{2}$, we obtain

$$
\left\{\begin{array}{c}
\lambda_{1}=\frac{4+2 \mu}{2+4 \mu+\mu^{2}} \\
\lambda_{2}=\frac{1}{2} \frac{2+4 \mu+\mu^{2}}{8+33 \mu+13 \mu^{2}}
\end{array}\right.
$$

which yields

$$
\alpha=\frac{(2+\mu)^{2}}{8+33 \mu+13 \mu^{2}} .
$$

\section{CONCLUSION}

This paper has established three main results: a regularity result for the continuous Galerkin formulation for the saturation equation, a regularity result for a linear scheme for the saturation equation, and error estimates for a fully discretized and linearized scheme for the same equation, under reasonable conditions on the data, and without any pretention to optimal estimates. The choices made here are not necessarily optimal, but they do show there is convergence when the perturbation, spatial discretization, and time-stepping parameters, $\beta, h$, and $\Delta t$ are chosen in such away that all converge to 0 , with the first two as some powers of the latter. How good is the proposed method will hopefully come from a continuation of this work which will concern itself with improving the present results by improving, for instance, Lemma 2.4 above, and, especially, doing some numerical experiments. The results obtained in this paper show, as expected, that we have a higher rate of convergence for the non degenerate case, i.e. for the case $k(s) \geq k_{0}>0$, for all $s \in[0,1]$. Another sequel of this paper will look more in depth at the special case of nondegenerate problems like the concentration problem.

The author intends to pursue investigations in the $H^{1}$ norm i.e. investigations for estimates for

$$
\sum_{0 \leq n \leq N} \Delta t\left\|\nabla\left(U_{h}^{n+1}-V_{h}^{n+1}\right)\right\|_{L^{2}}^{2} .
$$

As stated above, our perspective for a next paper (in association with other authors) is to implement effectively the scheme (3.3)-(3.4) on a computer and compare our results with the present estimates, and with existing methods.

\section{ACKNOWLEDGEMENTS}

The author would like to thank the referee for the useful comments and for pointing to works similar to the present paper. 


\section{CONFLICT OF INTERESTS}

Authors confirm that this article content has no conflicts of interest.

[11] element method for a degenerate parabolic equation arising in flow in porous media. SIAM J Numer Anal 1996; 33: 669-87.

[12] Rose ME. Numerical methods for flow through porous media-I. Math Comput 1983; 40: 437-67.

[13] Rose ME. Numerical methods for flow through porous media-II Comput Math Appl 1980; 6: 99-122.

[14] Fadimba KB. Error estimates for a regularization of a class of porous medium equations. Imhotep, J Math Pures Appl 2000; 3: 77-96.

\section{REFERENCES}

[1] Bear J, Verruijt A. Modeling groundwater flow and pollution. Dodreich, Holland: D. Reidel Publication Company 1987.

[2] Chavent G, Jaffre J. Mathematical models and finite element for reservoir simulation: single phase, multiphase and multicomponent flows through Porous Media. New York: North-Holland 1986.

[3] Ewing RE. Problems arising in the modeling of processes for hydrocarbon recovery. In: Ewing RE, Ed. The Mathematics of reservoir simulation. Philadelphia: SIAM 1983: pp. 3-34.

[4] Peaceman DW. Fundamentals of numerical reservoir simulation. New York: Elsevier Scientific Publishing Company 1977.

[5] Chen Z, Ewing RE. Degenerate two-phase incompressible flow-III: sharp error estimates. Numer Math 2001; 90: 215-40.

[6] Fadimba KB. A Linearization of a backward Euler scheme for a class of degenerate nonlinear advection-diffusion equations. Nonlinear Anal 2005; 63(5-7): e1097-106.

[7] Fadimba KB. A linear backward Euler scheme for the saturation equation: regularity results and consistency. J Comput Appl Math 2010; 234: 272-82.

[8] Fadimba KB. Regularization and numerical methods for a class of porous medium equations. Columbia: University of South Carolina 1993.

[9] Fadimba KB, Sharpley RC. A priori estimates and regularization for a class of porous medium equations. Nonlinear World 1995; 2: 13-41.

[10] Fadimba KB, Sharpley RC. Galerkin finite element method for a class of porous medium equations. Nonlinear Anal Real World Appl 2004; 5: 355-87.
[15] Smylie DL. A near optimal order approximation to a class of twosided nonlinear parabolic partial differential equations. Laramie: University of Wyoming 1989.

[16] Pop IS, Radu F, Knabner P. Mixed finite elements for the Richards' equation: linearization procedure. J Comput Appl Math 2004; 168 365-73.

[17] Pop IS. Error estimates for a time discretization method for the Richards' equation. Comput Geosci 2002; 6: 141-60.

[18] Radu FA, Knabner P, Pop IS. Error estimates for a mixed finite element discretization of some degenerate parabolic equations. Numerische Mathematik 2008; 109: 285-311.

[19] Klausen RA, Radu FA, Eigestad GT. Convergence of MPFA on triangulations and for Richards' equation. Int J Numer Method Fluids 2008; 58: 1327-51.

[20] Nochetto RH, Verdi C. Approximation of degenerate parabolic problems using numerical integration. SIAM J Numer Anal 1988; 25: 784-814

[21] Celia MA, Bouloutas ET, Zarba RL. A general mass-conservative numerical solution for the unsaturated flow equation. Water Resour Res 1990; 26(7): 1483-96.

[22] Brenner SC, Scott LR. The mathematical theory of finite element methods. Texts in Applied Mathematics 15. Berlin: Springer 1994.

[23] Adams RA. Sobolev spaces. New York: Academic Press 1975.

[24] Dautray R, Lions JL. Mathematical analysis and numerical methods for science and technology. Berlin: Springer-Verlag 1988; vol. 2.

(C) Koffi B. Fadimba; Licensee Bentham Open.

This is an open access article licensed under the terms of the Creative Commons Attribution Non-Commercial License (http://creativecommons.org/licenses/by$\mathrm{nc} / 3.0 /$ ) which permits unrestricted, non-commercial use, distribution and reproduction in any medium, provided the work is properly cited. 